\title{
Particle Based Simulation for Solitary Waves Passing over a Submerged Breakwater
}

\author{
Meng-Yu Lin*, Chiung-Yu Li, An-Pei Wang \\ Department of Civil Engineering, Chung Yuan Christian University, Chung Li 32023 \\ Email: ${ }^{*}$ mylin@cycu.edu.tw
}

Received January 2014

\begin{abstract}
This research develops a two-dimensional numerical model for the simulation of the flow due to a solitary wave passing over a trapezoidal submerged breakwater on the basis of generalized vortex methods. In this method, the irrotational flow field due to free surface waves is simulated by employing a vortex sheet distribution, and the vorticity field generated from the submerged object is discretized using vortex blobs. This method reduces the difficulty in capturing the nonlinear deformation of surface waves, and also concentrates the computational resources in the compact region with vorticity. This numerical model was validated by conducting a set of simulations for irrotational solitary waves and then compared with the results of a relevant research. The comparisons exhibit good agreement. The rotational flows induced by different incident wave height were simulated and analyzed to study the effect of vorticity on the deformation and the breaking of solitary waves.
\end{abstract}

Keywords

Solitary Wave, Submerged Breakwater, Vortex, Vortex Method, Particle Simulation

\section{Introduction}

The interaction of surface water waves with submerged structures has attracted attention in many fields of engineering applications. Numerous investigations for this problem have been implemented based on potential-flow theory with the assumption that the flow is irrotational. For example, [1] employed a boundary element method to establish a two-dimensional numerical model for the simulation of surface waves in an irrotational, inviscid fluid flow, and then simulated the breaking of solitary waves passing over a trapezoidal submerged breakwater. One of the benefits of the approaches using the irrotational-flow assumption is the efficient computation by applying boundary integral methods, and these approaches usually predict the transformation of water waves accurately if flow separation is not severe. For many engineering problems the effects of flow separation should not be ignored; therefore, the studies using potential-flow theory usually only investigate the scattering of surface waves (see, e.g., [2]-[4]).

This research develops a two-dimensional numerical model for the simulation of the flow due to a solitary

*Corresponding author.

How to cite this paper: Lin, M.-Y., Li, C.-Y. and Wang, A.-P. (2014) Particle Based Simulation for Solitary Waves Passing over a Submerged Breakwater. Journal of Applied Mathematics and Physics, 2, 269-276.

http://dx.doi.org/10.4236/jamp.2014.26032 
wave passing over a trapezoidal submerged breakwater on the basis of generalized vortex methods. The main object of this research is to gain insight into the deformation of free surface as well as the shedding of vortices from the breakwater. In this study an essentially grid-free numerical model is developed. The motivation is based on the fact that in water-wave problems the flow field far from submerged bodies can be simplified to be irrotational and may be treated by including a viscous-flow correction to the potential-flow analysis. To accomplish this, we apply the Helmholtz decomposition to decompose the flow field into two components: one related to the vorticity (vortical velocity) and the other being irrotational. The potential flow with surface waves is solved by employing a generalized vortex sheet approach, and the viscous flow is solved via a Lagrangian vortex particle method. In contrast with grid-based schemes, the Lagrangian vortex particles convect without numerical dissipation and automatically adjust to resolve the regions with vorticity.

\section{Mathematical Formulations}

\subsection{Governing Equations}

The problem of interest concerns the calculation of the two-dimensional flow of a solitary wave over a near-wall circular cylinder in a uniform channel. The viscous effects as well as the generation of vorticity at the free surface are ignored. The $x$-axis lies in the undisturbed free surface and the $y$-axis points vertically upward with unit vectors $\hat{\mathbf{e}}_{x}$ an d $\hat{\mathbf{e}}_{y}$, respectively (see Figure 1). The corresponding fluid velocity components are $u$ and $v$ respectively, and $\mathbf{u}$ is the fluid velocity vector. The motion of the fluid is governed by the incompressible Navier-Stokes equations:

$$
\begin{gathered}
\nabla \cdot \mathbf{u}=0 \\
\frac{\mathrm{D} \mathbf{u}}{\mathrm{D} t}=\frac{\partial \mathbf{u}}{\partial t}+\mathbf{u} \cdot \nabla \mathbf{u}=-\frac{1}{\rho} \nabla p-g \hat{\mathbf{j}}+v \nabla^{2} \mathbf{u}
\end{gathered}
$$

where $p$ is the fluid pressure, $\rho$ is the density of fluid, $g$ is the gravitational acceleration, and $v$ is the kinematic viscosity. In velocity-vorticity form, above equations can be expressed by the vorticity transport equation:

$$
\frac{\mathrm{D} \omega}{\mathrm{D} t}=v \nabla^{2} \omega
$$

where $\omega=\hat{\mathbf{j}} \nabla \times \mathbf{u}$ is the vorticity field with the unit vector $\hat{\mathbf{j}}$ out of the page.

\subsection{Boundary Conditions}

At the free surface, the flow field satisfies the kinematic and dynamic boundary conditions, which can be expressed in a Lagrangian description as

$$
\frac{\mathrm{D} \mathbf{x}_{f}}{\mathrm{D} t}=\mathbf{u}\left(\mathbf{x}_{f}\right)
$$

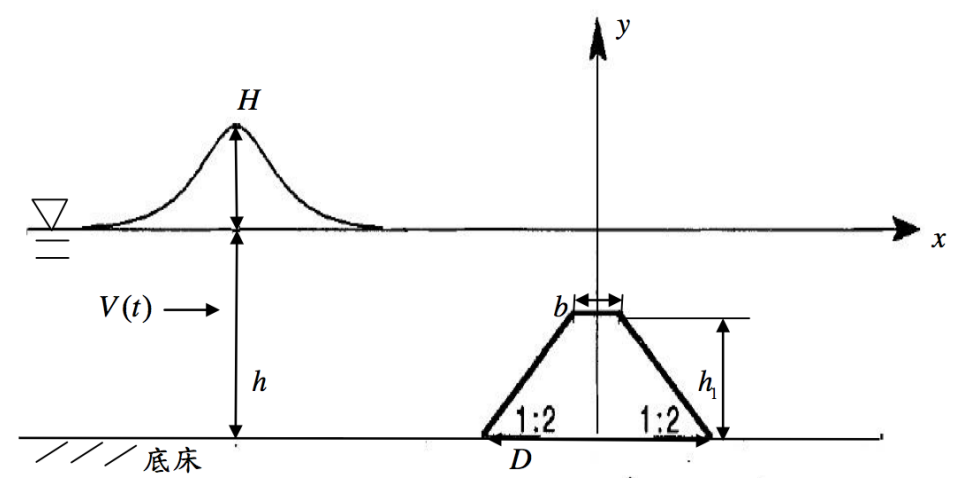

Figure 1. Definition sketch of the problem. 


$$
\frac{\mathrm{Du}}{\mathrm{D} t}\left(\mathbf{x}_{f}\right)=-\frac{1}{\rho} \nabla p_{a}-g \hat{\mathbf{j}}
$$

On solid boundaries (at the seabed or the boundary of the cylinder) $S_{b}$, no-slip boundary condition is employed:

$$
\mathbf{u}=\mathbf{0} \quad \text { on } \mathrm{S}_{b}
$$

\section{Generalized Vortex Methods}

If the interface between air and water is not perpendicular to the pressure gradient, vorticity will be generated and must be confined to the interface at all times because of the absence of free-surface viscosity. The so-called generalized vortex sheet approach for interfacial waves is the basis for several different computational methods [5]-[7].

The flow field studied in this research is generated by the free-surface waves and the vorticity separated from the circular cylinder and the seabed. By applying the integral formulations of the Helmholtz decomposition [8], the integral representation for the velocity field has the form:

$$
\mathbf{u}(\mathbf{x})=\int_{V} \mathbf{K}\left(\mathbf{x}-\mathbf{x}^{\prime}\right) \omega\left(\mathbf{x}^{\prime}\right) d \mathbf{x}^{\prime}+\int_{S_{f}} \mathbf{K}\left(\mathbf{x}-\mathbf{x}^{\prime}\right) \gamma\left(\mathbf{x}^{\prime}\right) d \mathbf{x}^{\prime},
$$

where $V$ is the volume of fluid, $S_{f}$ denotes the free surface, $\gamma$ is the strength of a vortex sheet at the free surface, and

$$
\mathbf{K}(\mathbf{x})=\frac{(-y, x)}{2 \pi|\mathbf{x}|^{2}}
$$

In (7), The first integral represents the rotational flow field induced by vorticity, and the second integral represents the irrotational flow field induced by the vortex sheet along the free surface. The vortex sheet strength $\gamma$ is related to the tangential velocity jump across the free surface:

$$
\gamma=\left(\mathbf{u}_{1}-\mathbf{u}_{2}\right) \cdot \hat{\mathbf{s}}
$$

where $\mathbf{u}_{1}$ and $\mathbf{u}_{2}$ are the limiting values of the velocity vector below and above the free surface, respectively, and $\hat{\mathbf{s}}$ is a unit vector oriented tangent to the vortex sheet (see Figure 2).

From the integral representation shown in (7) with the vorticity transport Equation (3) and the evolution equation for $\gamma$ derived from the dynamic free surface boundary condition (5), an alternative problem can be constructed for solving $x f, \omega$ and $\gamma$ rather than the original problem with primitive variables $x f, \mathrm{u}$ and $p$. The flow field evolves by following the trajectories of the vorticity-carrying elements $x \omega$ and the free-surface points $x f$, and then updating their strengths based on the following sets of equations. For vorticity $\omega$,

$$
\begin{gathered}
\frac{d \mathbf{x}_{\omega}}{d t}=\mathbf{u} \\
\frac{d \omega}{d t}=v \nabla^{2} \omega
\end{gathered}
$$

and for vortex sheet $\gamma$,

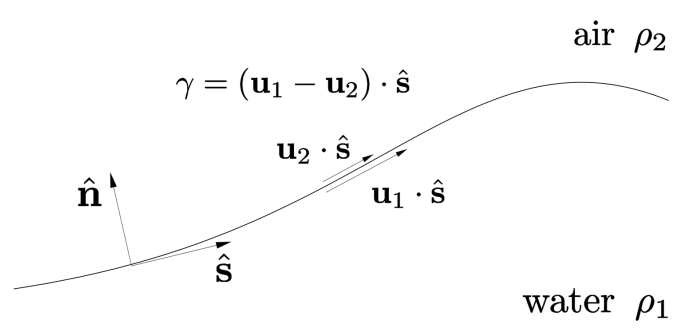

Figure 2. Vortex sheet along the free surface. 


$$
\begin{gathered}
\frac{d \mathbf{x}_{f}}{d t}=\mathbf{u}_{f} \\
\frac{d \gamma}{d t}=-\gamma \frac{\partial \mathbf{u}_{f}}{\partial s} \cdot \hat{\mathbf{s}}+2 \mathbf{a}_{f} \cdot \hat{\mathbf{s}}+2 g \hat{\mathbf{j}} \cdot \hat{\mathbf{s}}
\end{gathered}
$$

where $\mathbf{a}_{f}$ is the averaged velocity across the free surface, and

$$
\mathbf{a}_{f} \cdot \hat{\mathbf{s}}=\frac{d \mathbf{u}_{f}}{d t} \cdot \hat{\mathbf{s}}+\frac{1}{8} \frac{\partial\left(\gamma^{2}\right)}{\partial s}
$$

The vorticity field $\omega$ is determined by applying a Lagrangian vortex particle method to obtain a numerical approximation in terms of $N$ vorticity-carrying particles

$$
\omega(\mathbf{x}, t)=\sum_{j=1}^{N} \eta_{\epsilon}\left(\mathbf{x}-\mathbf{x}_{j}(t)\right) \Gamma_{j}(t)
$$

Each particle is identified by its position, $\mathbf{x}_{j}$ and its circulation, $\Gamma_{j}$. The regularized function $\eta_{\varepsilon}$ is the Gaussian distribution:

$$
\eta_{\epsilon}(\mathbf{x})=\frac{1}{2 \pi \epsilon^{2}} \exp \left(-\frac{|\mathbf{x}|^{2}}{2 \epsilon^{2}}\right)
$$

The velocity of the particle at $\mathbf{x}_{j}$ is

$$
\mathbf{u}\left(\mathbf{x}_{j}\right)=\sum_{i=1}^{N} \mathbf{K}_{\epsilon}\left(\mathbf{x}_{j}-\mathbf{x}_{i}\right) \Gamma_{i}+\int_{S_{f}} \mathbf{K}\left(\mathbf{x}_{j}-\mathbf{x}\right) \gamma(\mathbf{x}) d \mathbf{x}
$$

where

$$
\mathbf{K}_{\epsilon}(\mathbf{x})=\mathbf{K}(\mathbf{x})\left[1-\exp \left(-\frac{|\mathbf{x}|^{2}}{2 \epsilon^{2}}\right)\right]
$$

\section{Model Validation}

To validate the present model, we simulate the flow problem that was investigated in [1] using a boundary element method, and then compare the results of two computations. The simulated region is shown in Figure 1, where $H$ is the wave height, $h$ the still-water depth, $D$ and $b$ the width of the bottom and the top of the breakwater, respectively, and $h_{1}$ the height of the breakwater. In all computations $b=0.2 h, D=0.4 h$ and $h_{1}=0.8 h$. The cases of relative wave height $H / h=0.06,0.1,0.2,0.3,0.4$ and 0.5 are simulated. Since the simulations in [1] ignore viscous effects, in the computations using the proposed model presented in this section the effects of viscosity and the generation of vorticity are also ignored.

Figure 3 shows the computational results in [1] for the evolution of the free surface passing over the breakwater under different wave height, and Figure 4 is the corresponding results computed by the present model. The evolution of the solitary wave in each case predicted by the present model is seen to be in fairly agreement with the results in [1]. The small discrepancy between two simulations is because that two models employ different method to generate the solitary wave.

\section{Results and Discussion}

Figure 5 shows the vorticity fields at indicated times for $H / h=0.4$. When the wave crest is on the top of the breakwater $(t=14)$, the shear layers at the left and the right side of the breakwater separate from the wall and then generate vortices into the flow. When the wave crest is leaving $(t=16)$, the vortices at the left and right corner of the breakwater form recirculating regions and then induce secondary vortices on the wall. When the wave crest moves away from the breakwater $(t=18)$, the vortices dissipate gradually due to viscous effect because the driven flow disappears.

To investigate the effect of vorticity on the transformation of free surface, the comparison between the computed free surface transformation with and without vorticity effect is shown in Figure 6. Since the vortices 

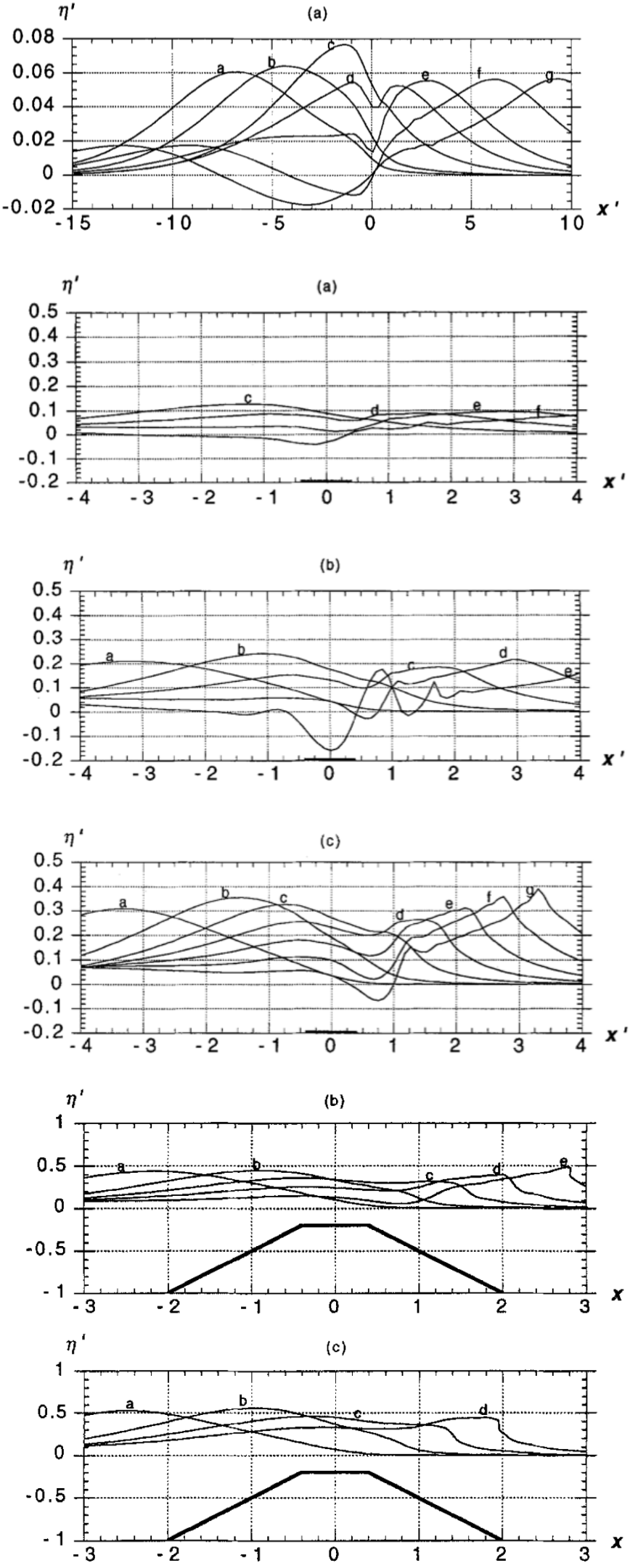

Figure 3. Computed results for solitary wave transformation in [1]. 
M.-Y. Lin et al.
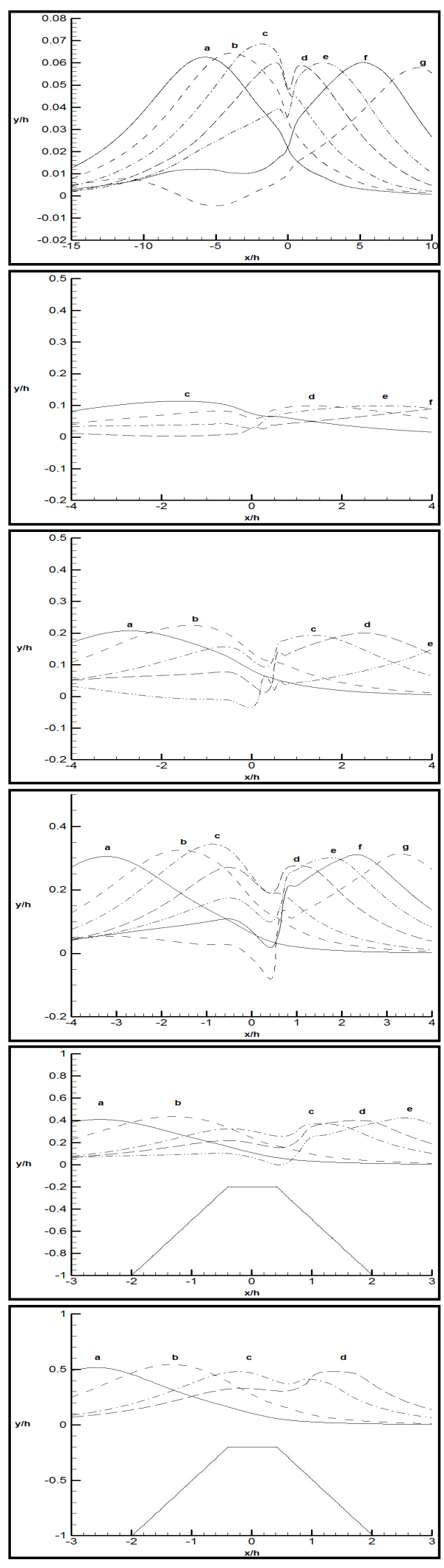

Figure 4. Solitary wave transformation commuted by the present model.

274 

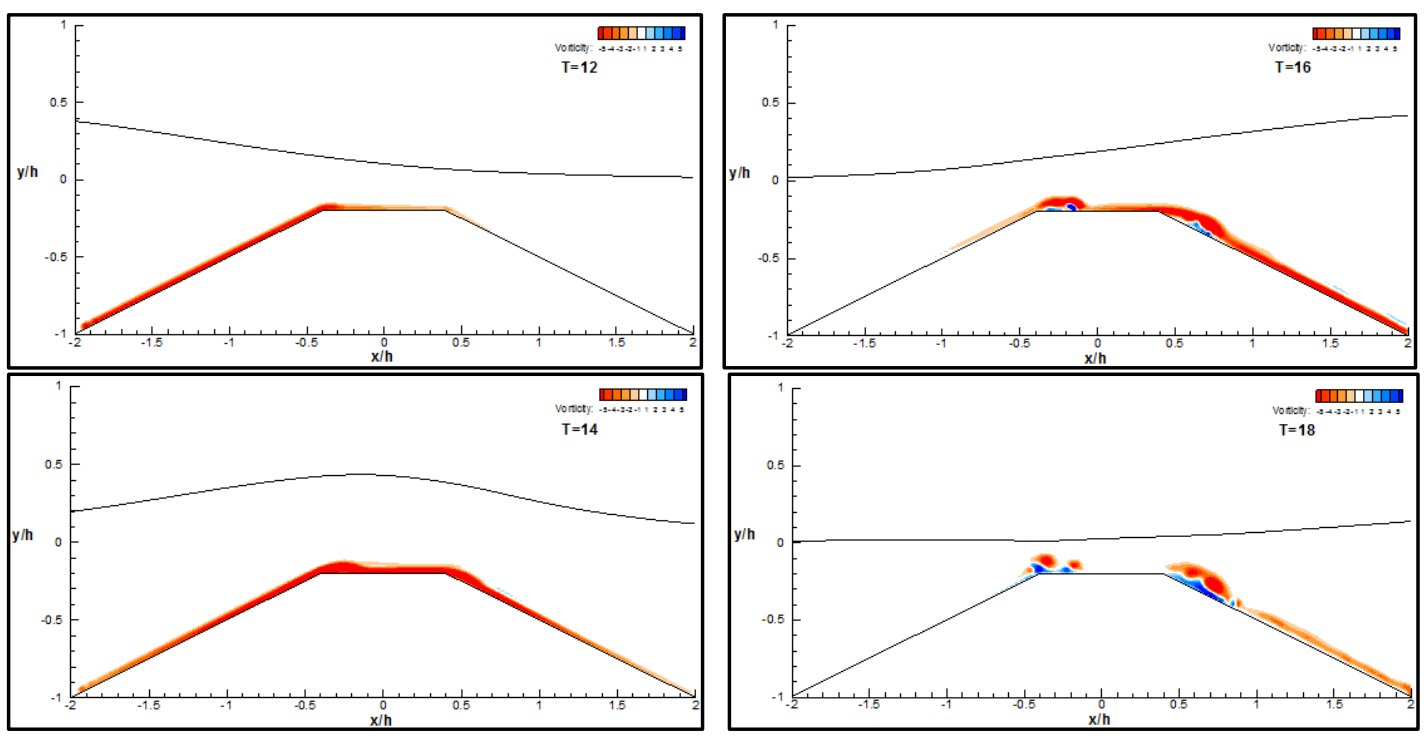

Figure 5. Vorticity fields at indicated times for $H / h=0.4$.

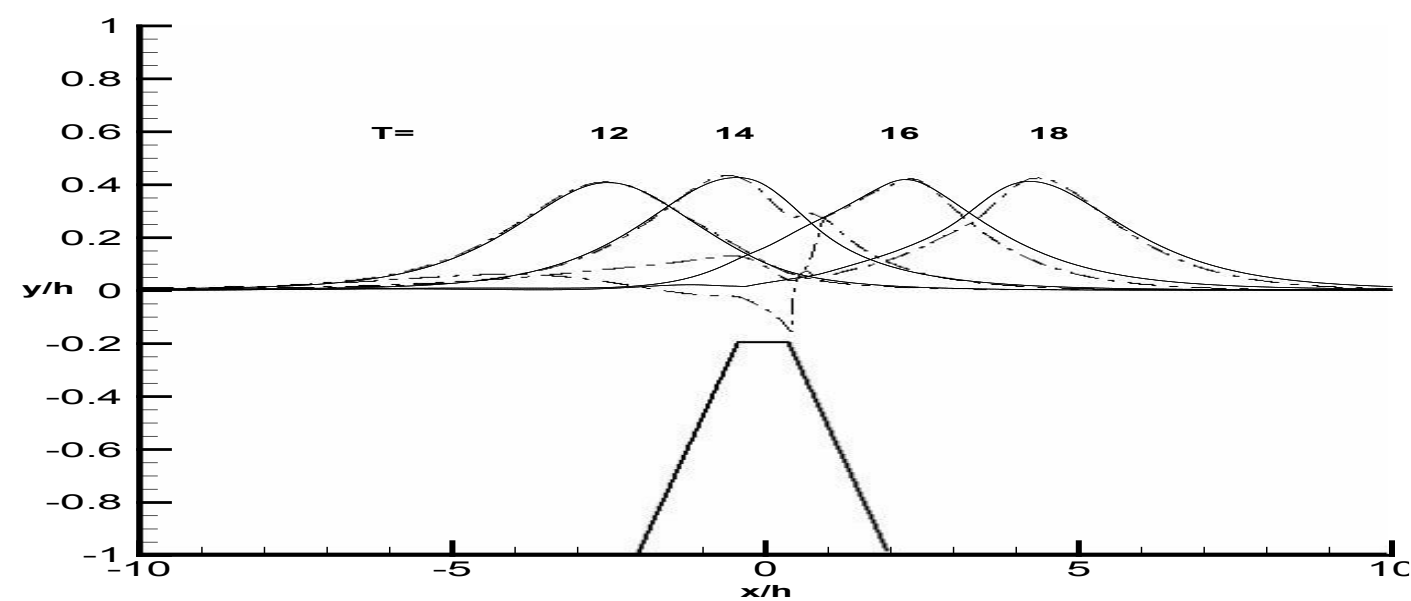

Figure 6. Comparison of free surface transformation for $H / h=0.4$ (-: rotational flow; - -: irrotational flow).

form recirculating regions on the wall of the breakwater, the severe deformation of free surface in the case of irrotational computation do not occur in the case with vorticity effect.

\section{References}

[1] Grilli, S.T., Losada, M.A. and Martin, F. (1994) Characteristics of Solitary Wave Breaking Induced by Breakwaters. Journal of Waterway Port Coastal and Ocean Engineering-ASCE, 120, 609-628. http://dx.doi.org/10.1061/(ASCE)0733-950X(1994)120:6(609)

[2] Yenug, R.W. and Vaidhyanathan, M. (1992) Non-Linear Interaction of Water Waves with Submerged Obstacles. International Journal for Numerical Methods in Fluids, 14, 1111-1130. http://dx.doi.org/10.1002/fld.1650140907

[3] Gobbi, M.F. and Kirby, J.T. (1999) Wave Evolution over Submerged Sills: Tests of High-Order Boussinesq Model. Coastal Engineering, 37, 57-96. http://dx.doi.org/10.1016/S0378-3839(99)00015-0

[4] Guyenne, P. and Nicholls, D.P. (2005) Numerical Simulation of Solitary Waves on Plane Slopes. Mathematics and Computers in Simulation, 69, 269-281. http://dx.doi.org/10.1016/j.matcom.2005.01.005

[5] Longuet-Higgins, M.S and Cokelet, E.D (1976) The Deformation of Steep Surface Waves on Water. I. A Numerical Method of Computation. Proceedings of the Royal Society of London. A. Mathematical and Physical Sciences, 350, 1-26. http://dx.doi.org/10.1098/rspa.1976.0092 
[6] Baker, G.R., Meiron, D.I. and Orszag, S.A. (1982) Generalized Vortex Methods for Free-Surface Flow Problems. Journal of Fluid Mechanics, 123, 477-501. http://dx.doi.org/10.1017/S0022112082003164

[7] Tryggvason, G. (1988) Numerical Simulations of the Rayleigh-Taylor Instability. Journal of Computational Physics, 75, 253-282. http://dx.doi.org/10.1016/0021-9991(88)90112-X

[8] Lin, M.-Y. and Huang, L.-H. (2009) Study of Water Waves with Submerged Obstacles Using a Vortex Method with Helmholtz Decomposition. International Journal for Numerical Methods in Fluids, 60, 119-148. http://dx.doi.org/10.1002/fld.1873 